\title{
DESIGN OF LOW LEVEL RF CONTROL FOR THE TESLA SUPERSTRUCTURE
}

\author{
S. Michizono ${ }^{\dagger}$, KEK, Tsukuba, Japan \\ M. Liepe, S. N. Simrock, DESY, Hamburg, Germany
}

\begin{abstract}
The superstructure is a viable option for the TESLA linear collider because of a high effective gradient and a reduced number of rf components. However, the high number and close proximity of passband modes impose challenging demands on the rf control system. The control problem is complicated by the fact that the cavity probe signal does not exactly reflect the actual accelerating voltage experienced by the beam due to the different coupling of the generator, pickup probe and beam to the FM passband modes. The digital control system developed for the standard 9-cell cavity is not adequate for operation of the superstructure. As discussed in this paper, an additional filter is needed to guarantee robustness and stability. Based on rf control simulations the filter characteristic is optimized.
\end{abstract}

\section{INTRODUCTION}

The performance of the superstructure [1] is planned to be tested with beam at the TESLA test facility (TTF). The main advantages of using superstructures are their higher space efficiency and fewer rf components. However, the superstructure (4x7-cell cavity) has 28 modes (fundamental acceleration mode is 25 th mode). Since the nearest mode $\left(26^{\text {th }}\right)$ is located only about $150 \mathrm{kHz}$ above the operation frequency $(1.3 \mathrm{GHz})$, careful LLRF control is important.

In the TTF LLRF system, the down-converter (250 $\mathrm{kHz}$ ) is used to detect the Inphase (I) and Quadrature (Q) components of the cavity field [2]. The signal obtained every $1 \mu$ s corresponds to one of I, Q -I and -Q. In order to reduce the excitation of the $26^{\text {th }}$ mode, a bandpass filter (BPF) with the center frequency of $250 \mathrm{kHz}$ (the carrier frequency) is inevitable for stable operation. The amplitude and phase errors are required to be less than

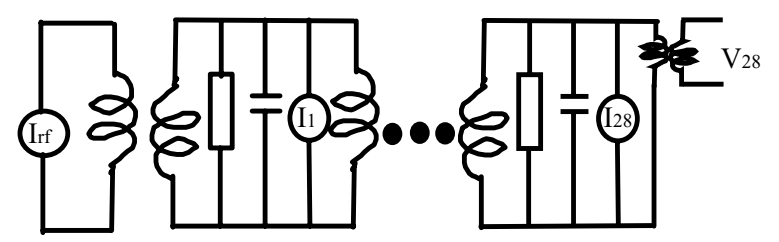

Figure 1 Schematic of the cavity model by a LCR circuit. Each cell couples with the adjacent cell. $\mathrm{I}_{\mathrm{rf}}$ represents the generator drive while $\mathrm{I}_{1}, \ldots \mathrm{I}_{28}$ describe excitation of the individual cells by the beam.
$0.5 \%$ and 0.5 degree, respectively [3].

\section{BASIC EQUATIONS FOR LLRF}

The calibrated cavity probe voltage $\left(\mathrm{V}_{\text {probe }}\right)$ is measured through a coupler adjacent to the $28^{\text {th }}$ cell (last cell), as schematically shown in Figure 1. In the following the calibrations are such that $\mathrm{V}_{\text {probe }}(25)$ equals the beamacceleration voltage $\mathrm{V}_{\text {acc }}(25)$ for the $25^{\text {th }}$ mode (accelerating mode). Although the electron beam $\left(\mathrm{I}_{\mathrm{b}}\right)$ excites the modes slightly (except $25^{\text {th }}$ mode), the $\mathrm{rf}$ source $\left(I_{\mathrm{rf}}\right)$ having about $20 \mathrm{MHz}$ bandwidth [2] can excite all of the modes.

The different couplings of the generator and the beam to the 28 passband modes are described by $r / q(n)$ and $\mathrm{R} / \mathrm{Q}(\mathrm{n})$ respectively. The cavity excitation is given by:

$$
\ddot{V}_{\text {probe }}+\frac{\omega_{0}}{Q_{l}} \dot{V}_{\text {probe }}+\omega_{0}^{2} V_{\text {probe }}=\frac{\omega_{0}}{2} \frac{r}{q} \dot{I}_{r f}-\frac{\omega_{0}}{2} \frac{R}{Q} \dot{I}_{b}
$$

Suppose that the stored energy of each mode is unity,

$$
U(n)=1=\frac{v_{\text {probe }}^{2}(n)}{\omega_{0}(n) \frac{r}{q}(n)}=\frac{v_{\text {probe }}^{2}(25)}{\omega_{0}(25) \frac{R}{Q}(25)} .
$$

Thus, the $\mathrm{r} / \mathrm{q}$ is written as

$$
\frac{r}{q}(n)=\frac{\omega_{0}(25)}{\omega_{0}(n)} \frac{v_{\text {probe }}^{2}(n)}{v_{\text {probe }}^{2}(25)} \frac{R}{Q}(25) \text {, }
$$

where $v_{\text {probe }}(\mathrm{n})$ and $v_{\text {probe }}(25)$ are the "unit" cavity probe voltage corresponding to the unity energy of the $n^{\text {th }}$ and $25^{\text {th }}$ modes, respectively.

By introducing the 'unit' beam acceleration voltage $\left(v_{\text {acc }}\right)$ corresponding to the unit stored energy,

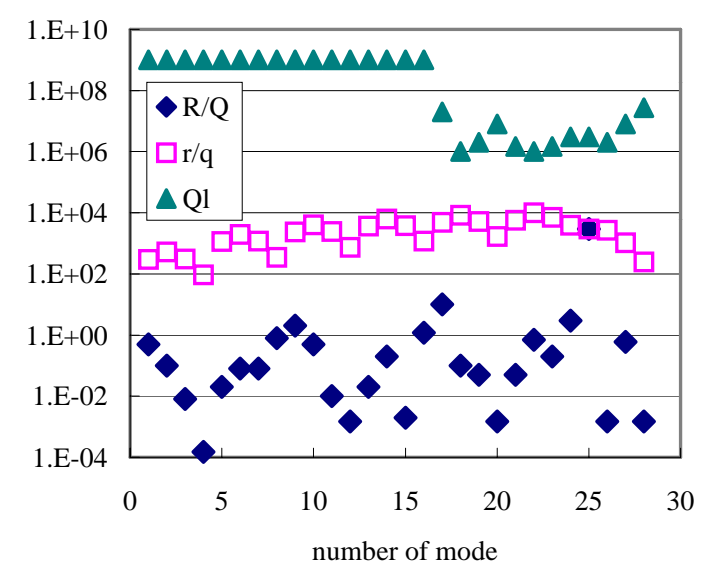

Figure 2 r/q distribution of each mode. R/Q and $\mathrm{Q}_{1}$ are referred from [1]. 


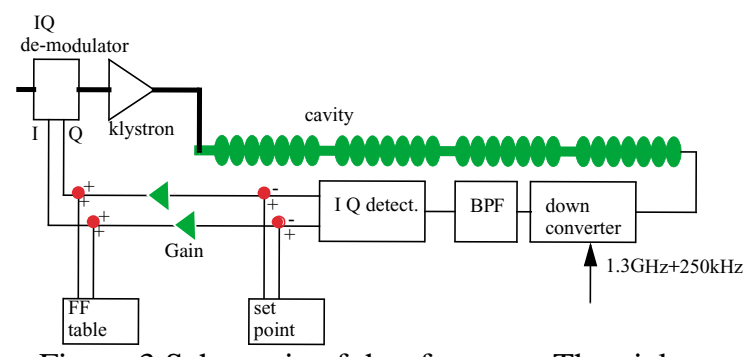

Figure 3 Schematic of the rf system. The pick-up signal of the cavity probe voltage is down-converted

to $250 \mathrm{kHz}$. A bandpass filter is used for reducing the adjacent $26^{\text {th }}$ mode.

$$
U(n)=1=\frac{v_{\text {probe }}^{2}(n)}{\omega_{0}(n) \frac{r}{q}(n)}=\frac{v_{a c c}^{2}(n)}{\omega_{0}(n) \frac{R}{Q}(n)} .
$$

Thus, the relation between the beam-acceleration voltage and the probe voltage is expressed as

$$
\frac{V_{\text {acc }}(n)}{V_{\text {probe }}(n)}=\sqrt{\frac{\frac{R}{Q}(n)}{\frac{r}{q}(n)}}=\sqrt{\frac{\omega_{0}(n) \frac{R}{Q}(n)}{\omega_{0}(25) \frac{R}{Q}(25)} \frac{v_{\text {probe }}(25)}{v_{\text {probe }}(n)}} .
$$

The calculated r/q are shown in Figure 2 together with $\mathrm{R} / \mathrm{Q}$ and $\mathrm{Q}_{1}$ referred from [1].

The relation between $\mathrm{V}_{\text {probe }}$, the beam current $\left(\mathrm{I}_{\mathrm{b}}\right)$ and the rf source equivalent current $\left(I_{\mathrm{rf}}\right)$ is written as follows:

$$
\begin{aligned}
& \frac{d}{d t}\left[\begin{array}{l}
V_{r} \\
V_{i}
\end{array}\right]=\left[\begin{array}{cc}
-\omega_{1 / 2}(n) & -\Delta \omega(n, t) \\
\Delta \omega(n, t) & -\omega_{1 / 2}(n)
\end{array}\right]\left[\begin{array}{c}
V_{r} \\
V_{i}
\end{array}\right] \\
& +\frac{\omega_{0}(25)}{2} \frac{R}{Q}(25) \frac{v^{2}{ }_{\text {probe }}(n)}{v^{2} \text { probe }(25)}\left(\left[\begin{array}{c}
I_{r f_{-} r} \\
I_{r f_{-}}
\end{array}\right]-\left(\frac{V_{\text {acc }}(n)}{V_{\text {probe }}(n)}\right)\left[\begin{array}{l}
I_{b_{-r}} \\
I_{b_{-i}}
\end{array}\right]\right) \text {, } \\
& \omega_{1 / 2}(n)=\frac{\omega_{0}(n)}{2 Q_{\ell}(n)}, \\
& \Delta \omega(n, t)=\omega_{c a v}(n, t)-\omega_{r f}=\omega_{c a v}(n, t)-2 \pi \cdot 1.3 e 9 .
\end{aligned}
$$

Here $\mathrm{V}_{\mathrm{r}}$ and $\mathrm{V}_{\mathrm{i}}$ are the real and imaginary parts of the probe voltage, respectively. Equation (6) means that the beam effect is smaller than that by the rf input.

\section{CALCULATION RESULTS}

Table 1: Typical parameters for the calculation.

\begin{tabular}{|c|c|}
\hline RF pulse & $1.6 \mathrm{~ms}$ \\
\hline Beam pulse & $\begin{array}{c}1 \mathrm{~ms} \\
(0.6 \mathrm{~ms}-1.6 \mathrm{~ms})\end{array}$ \\
\hline Beam & $8 \mathrm{nC} 1 \mathrm{MHz}$ \\
\hline R/Q (fundamental) & 2998 \\
\hline R/Q (other modes) & 10 \\
\hline BPF type & $4^{\text {th }}$ Bessel \\
\hline Loop delay & $5 \mathrm{micro} \mathrm{s}$ \\
\hline Feedback gain & $50(34 \mathrm{~dB})$ \\
\hline Beam acc. set voltage & $81 \mathrm{MV}[1]$ \\
\hline $\begin{array}{c}\text { Lorentz force } \\
\text { detuning constant }\end{array}$ & $1\left(\mathrm{~Hz} /(\mathrm{MV} / \mathrm{m})^{2}\right)$ \\
\hline
\end{tabular}

A calculation of the system was carried out with the parameters listed in Table 1. The schematic of the system is shown in Figure 3. The beam R/Q are set to 10 to estimate the worst conditions for control. The system stability is examined as function of the BPF bandwidth and analysed by use of Bode plots.

\subsection{BPF bandwidth}

The results with the parameter of the BPF bandwidth are shown in Figure 4. BPF bandwidth narrower than 150 $\mathrm{kHz}$ can sufficiently reduce the $26^{\text {th }}$ mode. The bandwidth narrower than $50 \mathrm{kHz}$ results in a slower response time, leading to poor control. Because the BPF with bandwidth wider than $150 \mathrm{kHz}$ permits excitation of the $26^{\text {th }}$ mode, the fundamental signal is not stable. The $250 \mathrm{kHz}$ downconverter system with optimised BPF enables us to control the fundamental mode.

\subsection{Microphonics}

In order to analyze the effect of microphonics the feedforward table obtained for a given Lorentz-force detuning curve is also applied for additional $+50 \mathrm{~Hz}$ and $-50 \mathrm{~Hz}$ detuning as induced by microphonics. Despite the feedforward table mismatch the regulation quality and loop stability do not suffer. The results are summarized in Table 2.

\subsection{Bode plot}

The system performance is compared with the standard 9-cell cavity system by the Bode plot as shown in Figure 5. Since the $27^{\text {th }}$ mode is located about $370 \mathrm{kHz}$ higher than the fundamental mode, the alias signal $(130 \mathrm{kHz})$ is shown. It is confirmed that the wide-bandwidth BPF (200 $\mathrm{kHz}$ ) cannot suppress the 26th mode sufficiently and that the narrow-bandwidth BPF $(25 \mathrm{kHz})$ results in low gainmargin due to the loop-phase shift.

The gain margin and pseudo-gain margins for the other

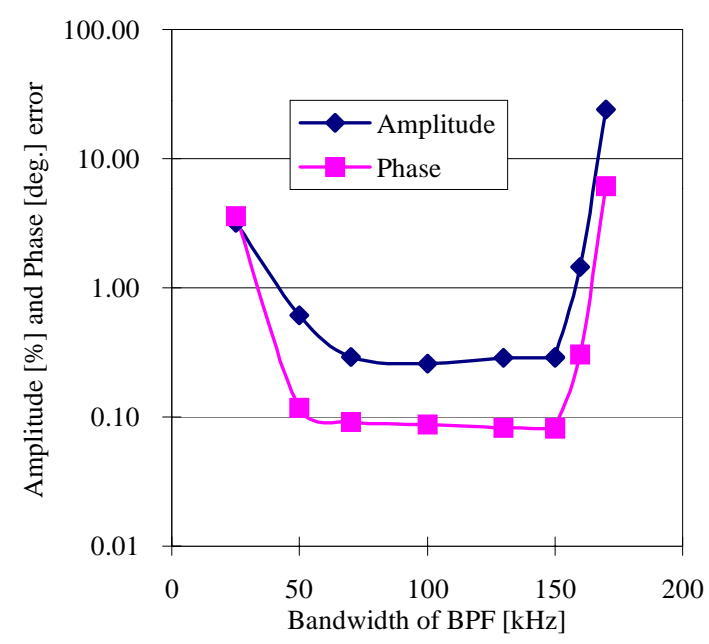

Figure 4 Dependence on the filter bandwidth. The center frequency of the BPF is $250 \mathrm{kHz}$. The errors become minimum for $50 \mathrm{kHz}$ to $150 \mathrm{kHz}$ bandwidth. 
Table 2: Calculation results for mismatched feedforward tables

\begin{tabular}{|l|c|c|}
\hline & $\begin{array}{c}\text { Amplitude } \\
\text { Error }\end{array}$ & $\begin{array}{c}\text { Phase } \\
\text { Error }\end{array}$ \\
\hline $0 \mathrm{~Hz}$ det. by microphonics & $0.26 \%$ & $0.09 \mathrm{deg}$. \\
\hline$+50 \mathrm{~Hz}$ detuning & $0.32 \%$ & $0.09 \mathrm{deg}$. \\
\hline$-50 \mathrm{~Hz}$ detuning & $0.27 \%$ & $0.09 \mathrm{deg}$. \\
\hline
\end{tabular}

modes, which are only the difference between $0 \mathrm{~dB}$ and peak amplitude without consideration of loop phase, are plotted in Figure 6. Although the real gain margin is different from the pseudo ones, the values are the safety ones because the phase would change due to the system delay. Half of the gain margin represents the gain limit for the feedback operation [2]. The results show that the maximum feedback gain with $70 \mathrm{kHz}$ bandwidth BPF is $38.5 \mathrm{~dB}$. This is about a quarter of the standard TTF control system $(=44 \mathrm{~dB})$. However, the gain is still sufficient to reduce the residual errors within the specifications.

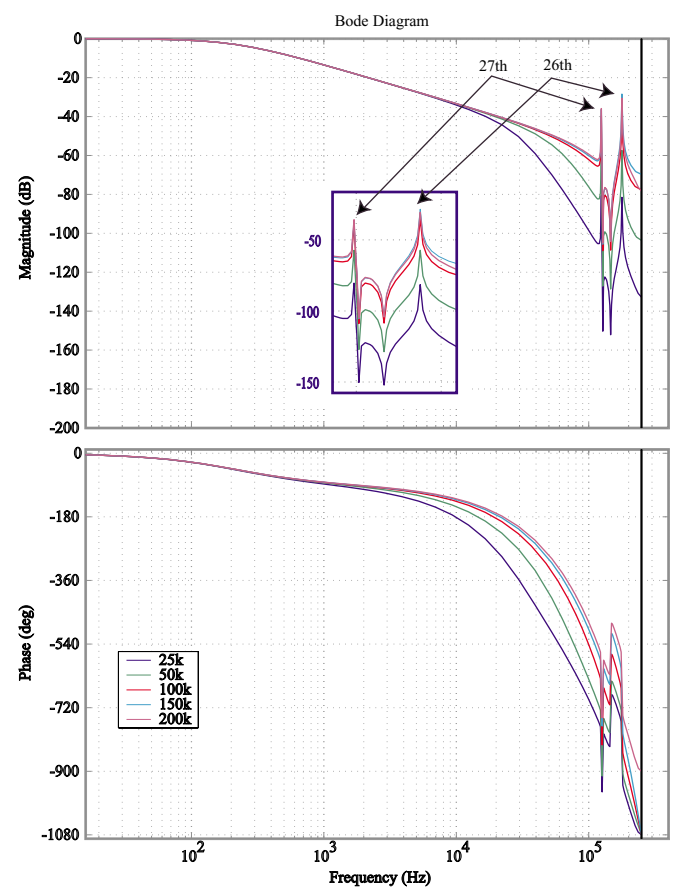

Figure 5 Bode plot for the superstructure cavity with BPF of $25 \sim 200 \mathrm{kHz}$ bandwidth. The alias $(130 \mathrm{kHz})$ of the $27^{\text {th }}$ mode located $370 \mathrm{kHz}$ higher than fundamental mode is shown in the figure.

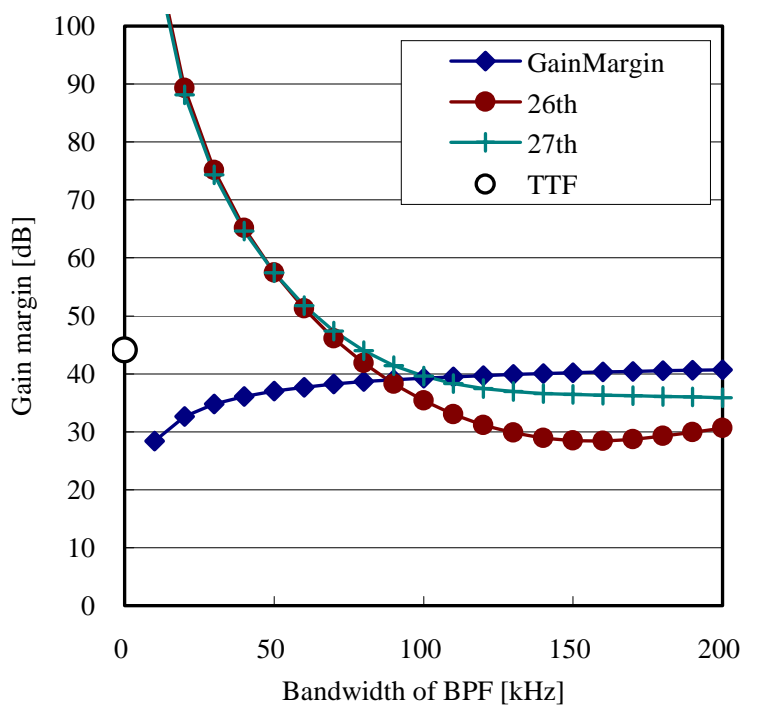

Figure 6 BPF bandwidth dependence on the operational gain. The pseudo-gain margins for the $26^{\text {th }}$ and $27^{\text {th }}$ modes are defined from the gain of the amplitude without considering the phase delay. "TTF" is the gain margin for the p-mode of the standard 9-cell TTF cavity.

\section{SUMMARY}

The LLRF system presently in operation at the TESLA Test Facility can be used for the superstructure by insertion of a bandpass filter at the IF output of the down converter. The simulation results demonstrate that performance goals are met for a wide range of loop gain and filter bandwidth. A loop gain of 50 and a filter bandwidth of $70 \mathrm{kHz}$ guarantees robustness against parameter variations.

\section{REFERENCES}

[1] J. Sekutowicz, M. Ferrario, and Ch. Tang, "Superconducting superstructure for the TESLA collider: A concept", Phys. Rev. ST Accel. Beams 2, 062001 (1999).

[2] T.Schilcher, "Vector Sum Control of Pulsed Accelerating Fields in Lorenz Force Detuned Superconducting Cavities", August 1998, TESLA 9820.

[3] S.N.Simrock, I.Altmann, K.Rehlich, T.Schilcher, "DESIGN OF THE DIGITAL RF CONTROL SYSTEM FOT THE TESLA TEST FACILITY", EPAC96, Sitges (Barcelona), June 10-14,1996, p.346. 